\title{
Immunoglobulin E-an Innocent Bystander in Host Defense?
}

\author{
Qian Zhang ${ }^{1} \cdot$ Mikko R. J. Seppänen ${ }^{2}$ (1) \\ Received: 31 January 2018 / Accepted: 4 February 2018 / Published online: 15 February 2018 \\ (C) Springer Science+Business Media, LLC, part of Springer Nature 2018
}

Discoveries of physiology often precede anatomy in immunology research. Forty-five years before the discovery of IgE, Prausnitz and Küstner found that allergy can be transferred by sera from allergic patients in 1921 [1]. Tested by erythemawheal assays, this serum fraction was named "reagin." With the advances in electrophoresis and ultracentrifugation between the 1930s and 1960s, IgG, IgM, IgA, and IgD were sequentially discovered. By the end of 1964, most immunologists and allergists believed that reagin is an allergen-specific IgA antibody. Eventually, in 1966, Ishizaka et al. discovered the immunoglobulin responsible for allergy - IgE, named after the erythema-wheal. IgE was the last immunoglobulin discovered due to its over 1000 times lower blood concentration in healthy controls compared with the other immunoglobulin classes. Although scarce, IgE characteristically has rapid, potent, and broad effects through $\mathrm{Fe} \varepsilon \mathrm{RI}$ and $\mathrm{CD} 23$ on a wide range of cell types [2]. The evolutionary persistence of IgE suggests an important role in host defense [2]. IgE was shown to opsonize Schistosoma mansoni for killing by eosinophils, to induce strong responses of skin mast cells and basophils to arthropods bites, and to activate mast cells to release proteases that neutralize venoms [2]. In sharp contrast to the abundant studies on increased IgE levels in the context of allergy, the clinical implication of decreased IgE levels remain largely neglected. In this issue, Lawrence et al. report a frequent lack of $\operatorname{IgE}$ in addition to other immunoglobulins in the majority of common variable immunodeficiency (CVID) patients. This should ignite new research into host defense functions of IgE [3].

CVID is the most common severe primary immunodeficiency in the world, with prevalence as high as 3.8-7.7:100,000 [4]. The

Mikko R. J. Seppänen

mikko.seppanen@hus.fi

1 Translational Medicine, Sidra Medical and Research Center, Doha, Qatar

2 Rare Disease Center, Children's Hospital, and Adult Primary Immunodeficiency Outpatient Clinic, Inflammation Center, University of Helsinki and Helsinki University Hospital, Helsinki, Finland accurate differentiation of CVID from secondary antibody deficiency is particularly imperative because adequately substituted patients on average live some 30 years longer than historical controls. Giving IgG replacement therapy to for example asthmatics with steroid-induced hypogammaglobulinemia may cause severe side effects [5]. Despite a rather uniform phenotype, there are multiple and variable available diagnostic criteria for CVID $[3,4]$. Much of this diagnostic confusion stems from variable cutoffs for normalcy used for immunoglobulin classes $\mathrm{IgG}$, IgA, and IgM, as well as for specific antibodies, especially for polysaccharide responses against pneumococcal antigens [3-5]. Typically, CVID patients have low IgG, low IgA, and specific antibody deficiency. However, at least $1.3 \%$ of primary antibody-deficient patients present with only IgG and IgM deficiency together with suboptimal specific antibody responses [4]. Secondary antibody deficiency due to the use of agents like rituximab or glucocorticoids much more commonly presents with such a combination of low Ig classes. Differentiating primary and secondary antibody deficiencies is also imperative for various other purposes, such as for epidemiologic and genetic studies. Consequently, the search for differentiation markers has been a long struggle in the field. Currently, anti-pneumococcal antibody responses are the favored specific marker for primary CVID. However, the diagnostic use of anti-pneumococcal antibody responses is still largely limited to the same serotypes included in conjugated pneumococcal vaccines. Therefore, their results are difficult to interpret in previously vaccinated children and elderly people. As alternatives, anti-Salmonella antibody responses and serum allohemagglutinins have been used to help in the differential diagnosis of CVID. However, these sadly seem to perform suboptimally [5]. Recently, reduced levels of serum free light chains were also proposed to function as differentiation markers for CVID [6].

The report of low serum IgE in CVID patients is thus a valuable observation for clinical diagnosis. By using contemporary accredited methods traceable to WHO reference preparation 75/502, Lawrence et al. found that serum IgE was lower than $2 \mathrm{IU} / \mathrm{ml}$ in $75.6 \%$ of CVID patients tested. In fact, CVID was associated with very low IgE already over 45 years ago [7]. Its use for diagnostic purposes was at that time not explored 
further, likely since it did not differentiate between IgA deficiency patients with or without infection susceptibility [8]. Lawrence et al. further show that low levels of serum IgE are useful in differentiating primary and secondary antibody immunodeficiencies. Results from our cohort largely corroborate theirs: the prevalences of undetectable IgE were $79 \%$ for probable CVID, 38\% for possible CVID and 50\% for secondary hypogammaglobulinemia after lymphoma therapy (unpublished) [4]. Since the use of agents like rituximab and glucocorticoids cause typical changes into B cell differential, its use together with serum IgE levels could greatly aid in differentiating secondary hypogammaglobulinemia from primary CVID.

Besides its potential and far-ranging clinical implications, this report remains intriguing also for basic studies on IgE production in humans. In other antibody responses, germinal center B cells undergo clonal expansion, somatic hypermutation (SHM), class switch recombination (CSR), and generation of long-term memory B cells. This process is different for IgE-producing B cells. Upon activation and initial expansion, IgE-expressing B cells either rapidly transform into plasma cells or are subject to immediate apoptosis. The generation of high-affinity $\operatorname{IgE}$ occurs in a unique way, in which B cells undergo first IgM to IgG, and then IgG to IgE CSR, also known as the sequential CSR [2]. This unique process results in two features of IgE production: 1. Most of the high-affinity IgE originates from IgG (or IgA)-expressing $\mathrm{B}$ cell lineages; 2. IgE memory resides in IgG-expressing memory B cells. Although direct CSR exists in both human and mouse, more and more recent data showed that the majority of $\operatorname{IgE}$ in humans is produced through sequential CSR $[9,10]$. Therefore, decreased IgG and IgE levels might reflect the insufficient memory B cell pool observed in a large proportion of CVID patients. Moreover, low serum IgE together with low IgG might reflect a more general defect in the activity of activation-induced cytidine deaminase (AID). AID generates mutations in germinal center B cells to initiate both CSR and SHM. In the human constant heavy chain locus, heavy chain genes reside in the order of IGHM-IGHG3-IGHG1-IGHE1_psIgHA1-IGH $p$ s-IGHG2-IGHG4-IGHE-IGHA2. Interestingly, in CVID patients, the genes more distal from the heavy chain locus enhancer and regulatory regions were thus expressed more poorly, reflected in the increased IgG1/IgG4 ratio and extremely low IgE levels described by the authors. Therefore, defects in AID activity or impairments after CSR at the stage when the proximal heavy chains are cut out from the germline DNA could potentially explain the overall decrease in the production of immunoglobulins and memory B cells in CVID.

In addition to total serum IgE, the authors were unable to detect circulating antigen-specific IgE in CVID patients. This was observed despite the fact that in the USIDNET cohort the rates of self-reported and/or physician-diagnosed allergy were substantial, as discussed by the authors. Interestingly, clinical allergy together with positive responses to bronchial provocation tests with allergens has been reported in CVID patients with undetectable serum IgE [11]. How such "pseudoallergic" reactions could be maintained, or even worsened, in CVID patients after the onset of disease seems puzzling. Also, as intriguing as the low serum IgE levels reported in CVID patients are, we should not neglect to study those over $3 \%$ of controls with undetectable serum IgE reported here and previously $[3,12]$. These individuals, who presumably have normal levels of other classes of immunoglobulins and normal numbers of total B cells, will be the key to understanding the specific role of $\operatorname{IgE}$ in host defense. We thus hope that this report will eventually lead into better definitions for CVID, as well as trigger more research into the physiologic functions of IgE.

\section{Compliance with Ethical Standards}

Conflict of Interest The authors declare that they have no conflict of interest.

\section{References}

1. Ishizaka K, Ishizaka T. Identification of IgE. J Allergy Clin Immunol. 2016;137(6):1646-50.

2. Oettgen HC. Fifty years later: emerging functions of $\operatorname{IgE}$ antibodies in host defense, immune regulation, and allergic diseases. J Allergy Clin Immunol. 2016;137(6):1631-45.

3. Lawrence MG, Palacios-Kibler TV, Workman LJ, Schuyler AJ, Steinke JW, Payne SC, et al. Low serum IgE is a sensitive and specific marker for common variable immunodeficiency (CVID). J Clin Immunol. 2018;38(3). https://doi.org/10.1007/s10875-018-0476-0.

4. Selenius JS, Martelius T, Pikkarainen S, Siitonen S, Mattila E, Pietikäinen R, et al. Unexpectedly high prevalence of common variable immunodeficiency in Finland. Front Immunol. 2017;8:1190.

5. Schaballie H, Bosch B, Schrijvers R, Proesmans M, de Boeck K, Boon MN, et al. Fifth percentile cutoff values for antipneumococcal polysaccharide and anti-Salmonella typhi Vi IgG describe a normal polysaccharide response. Front Immunol. 2017;8:546.

6. Hanitsch LG, Sotzny F, Volk H-D, Scheibenbogen C, Wittke K. Serum Free Light Chains in CVID - a Marker for Differential Diagnosis. J Clin Immunol. 2018;38(2). https://doi.org/10.1007/ s10875-018-0478-y.

7. Stites DP, Ishizaka K, Fudenberg HH. Serum IgE concentrations in hypogammaglobulinaemia and selective IgA deficiency. Studies on patients and family members. Clin Exp Immunol. 1972;10(3):391-7.

8. Polmar SH, Waldmann TA, Balestra ST, Jost MC, Terry WD. Immunoglobulin $\mathrm{E}$ in immunologic deficiency diseases. I. Relation of IgE and IgA to respiratory tract disease in isolated IgE deficiency, IgA deficiency, and ataxia telangiectasia. J Clin Invest. 1972;51(2):326-30.

9. Xiong H, Dolpady J, Wabl M, Curotto de Lafaille MA, Lafaille JJ. Sequential class switching is required for the generation of high affinity IgE antibodies. J Exp Med. 2012;209(2):353-64.

10. Looney TJ, Lee JY, Roskin KM, Hoh RA, King J, Glanville J, et al. Human B-cell isotype switching origins of IgE. J Allergy Clin Immunol. 2016;137(2):579-86. e7

11. Agondi RC, Barros MT, Rizzo LV, Kalil J, Giavina-Bianchi P. Allergic asthma in patients with common variable immunodeficiency. Allergy. 2010;65(4):510-5.

12. Ferastraoaru D, Gross R, Rosenstreich D. Increased malignancy incidence in IgE deficient patients not due to concomitant common variable immunodeficiency. Ann Allergy Asthma Immunol. 2017;119(3):267-73. 